\title{
Prevention of Stroke with Closed-Cell Case Report Stent-in-Stent for Carotid Artery Stenosis Due to Stent Fracture
}

Yutaro Fuse, Hayato Tajima, Shigekazu Nakamura, Futoshi Kurimoto, and Kazuhiko Watanabe

Objective: Stent fracture is a risk factor for stroke. It has not been fully elucidated whether stent-in-stent procedures can effectively treat stent fractures.

Case Presentation: An 80-year-old man underwent carotid artery stenting (CAS) with an open-cell stent to treat asymptomatic right internal carotid artery (ICA) stenosis. Type III stent fracture occurred during CAS. Six months later, in-stent stenosis progressed on DSA. Repeat CAS with a closed-cell stent was performed. CT showed expansion of the narrowed lumen. The patient remained stroke-free and carotid artery restenosis did not occur for 3 years postoperatively. Conclusion: Repeat CAS with a closed-cell stent is a viable treatment option for stent fracture.

Keywords > carotid artery stenosis, restenosis, stent fracture, stent deformity, stroke prevention

\section{Introduction}

Stent fracture and deformation are complications of carotid artery stenting (CAS), as it can lead to in-stent stenosis and stroke. ${ }^{1)}$ The stent-in-stent technique has been tested for the treatment of stent fracture ${ }^{2,3)}$; however, its ability to prevent future strokes is unclear. The present study reports a case of successful stroke prevention for 3 years after repeat CAS for stent fracture with a closed-cell stent.

\section{Case Presentation}

An 80-year-old man was referred to our neurosurgery department for asymptomatic right internal carotid artery (ICA) stenosis detected on screening echocardiography during a hospital stay for acute myocardial infarction. The patient underwent CAS under local anesthesia. The results

Department of Neurosurgery, Handa City Hospital, Handa, Aichi, Japan

Received: December 26, 2020; Accepted: March 31, 2021 Corresponding author: Yutaro Fuse. Department of Neurosurgery, Handa City Hospital, 2-29, Toyocho, Handa, Aichi 475-8599, Japan Email: yutaro.fuse@gmail.com

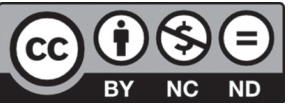

This work is licensed under a Creative Commons Attribution-NonCommercialNoDerivatives International License.

(C)2021 The Japanese Society for Neuroendovascular Therapy of preoperative DSA and CTA are depicted in Fig. 1. The diameter of right common carotid artery (CCA) was $7.2 \mathrm{~mm}$, that of right ICA was $4.9 \mathrm{~mm}$, and that of the lesion was $1.3 \mathrm{~mm}$. Prior to surgery, the patient was on dual antiplatelet therapy. An 8-Fr Optimo Balloon Guiding Catheter (Tokai Medical Products, Kasugai, Aichi, Japan) was placed in the right CCA using a right femoral artery approach. A Carotid GuardWire PS (Medtronic, Minneapolis, MN, USA) was then deployed to the right ICA for distal protection. The lesion was pre-dilated with a Genity $3.0 \times$ 40-mm (Kaneka Medix, Osaka, Japan). A Precise $10 \times 40 \mathrm{~mm}$ (Cordis, Santa Clara, CA, USA) was deployed over the ICA to the CCA. The lesion was then post-dilated with an Aviator Plus $4.0 \times 20$-mm balloon (Cordis). On DSA after post-dilation, stent fracture was observed (Fig. 2A). The stent lumen was not narrowed on DSA (Fig. 2B). The CAS procedure was finished without causing any neurological symptoms. Algatroban and ozagrel sodium were initiated and kept on the patient for 2 days. Dual antiplatelet therapy was continued thereafter. Postoperative MRI did not detect any brain infarction.

Six months later, follow-up DSA and cone-beam CT showed in-stent stenosis at the site of stent deformity (Fig. 3A-3C). The patient was free from strokes and transient ischemic attacks at that time. Repeat CAS was then performed on the patient. Informed consent was obtained for experimentation with human subjects. With the same approach as was used in the first procedure, an 8-Fr Optimo 


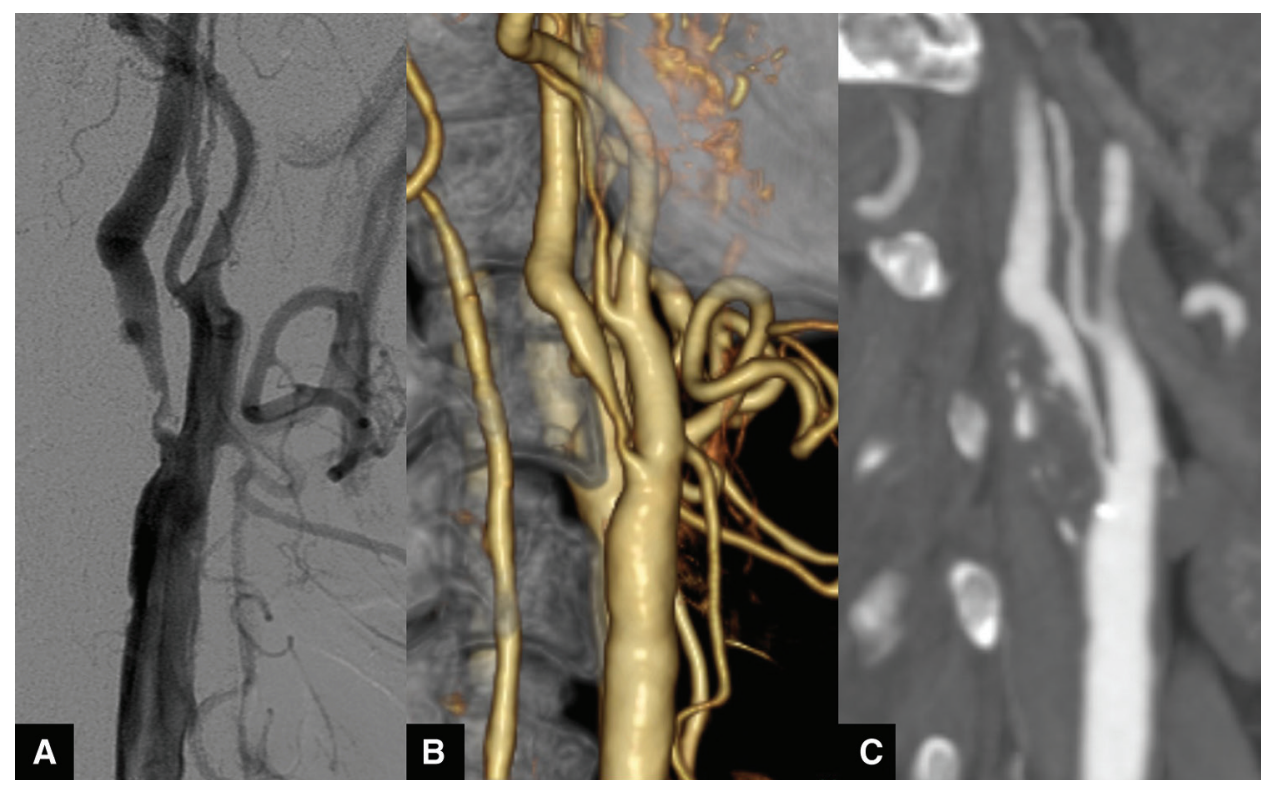

Fig. 1 Preoperative DSA (A) and CTA $(B, C)$ reveal stenosis of the ICA and the CCA. Calcification is observed at the site of stenosis (C). CCA: common carotid artery; ICA: internal carotid artery

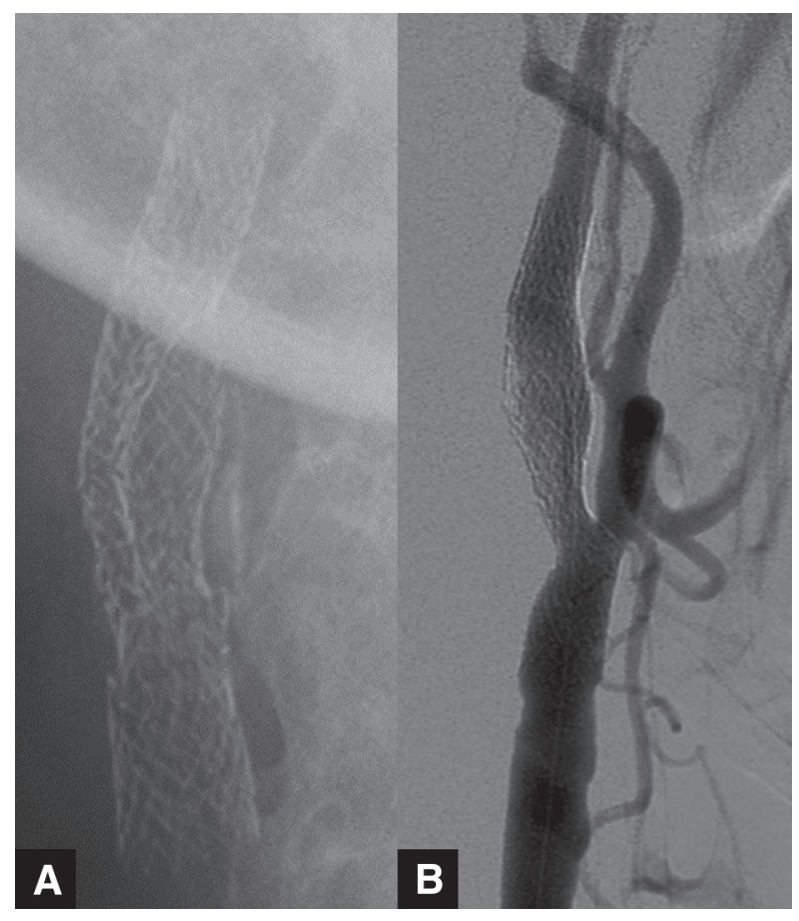

Fig. 2 Stent fracture is seen on postoperative X-ray (A). Improved blood flow on DSA (B).

Balloon Guiding Catheter was deployed to the CCA and a Guardwire was placed in the ICA. The diameter of right CCA was $7.5 \mathrm{~mm}$ and that of right proximal ICA was $6.5 \mathrm{~mm}$. The fractured stent is depicted in a $3 \mathrm{D}$ fashion (Fig. 3F). Balloon percutaneous transluminal angioplasty (PTA) was performed with an Aviator Plus $4.0 \times 20-\mathrm{mm}$ balloon, followed by a Genity $6.0 \times 20$-mm. In-stent stenosis did not improve after PTA. Therefore, a Wallstent $10 \times 24-\mathrm{mm}$ carotid stent (Wallstent; Boston Scientific, Natick, MA, USA) was deployed. Post-dilation was performed using a Genity $6.0 \times 20 \mathrm{~mm}$. Postoperative DSA cone-beam CT showed expansion of the narrowed lumen (Fig. 3D, 3E, and 3G). Algatroban was initiated and kept on the patient for 2 days. Dual antiplatelet therapy has been on the patient so far. Postoperative MRI did not detect any brain infarction. The patient's postoperative course was uneventful. The latest carotid artery duplex scan examined 3 years after repeat CAS did not show carotid artery restenosis (Fig. 4).

\section{Discussion}

In the current case of stent fracture, we identified two important clinical results. First, when stent deformity leads to in-stent stenosis, repeat CAS is effective to prevent strokes in the medium term. Second, a closed-cell stent is a useful device that can be used to expand the narrowed lumen after stent fracture.

The stent-in-stent technique is effective for stroke prevention in patients with progressive carotid artery restenosis caused by stent fracture. Stent fracture and deformity are not uncommon CAS device complication. ${ }^{4,5)}$ Although a randomized trial did not identify an association between stent fracture and stroke, ${ }^{6}$ other publications have 


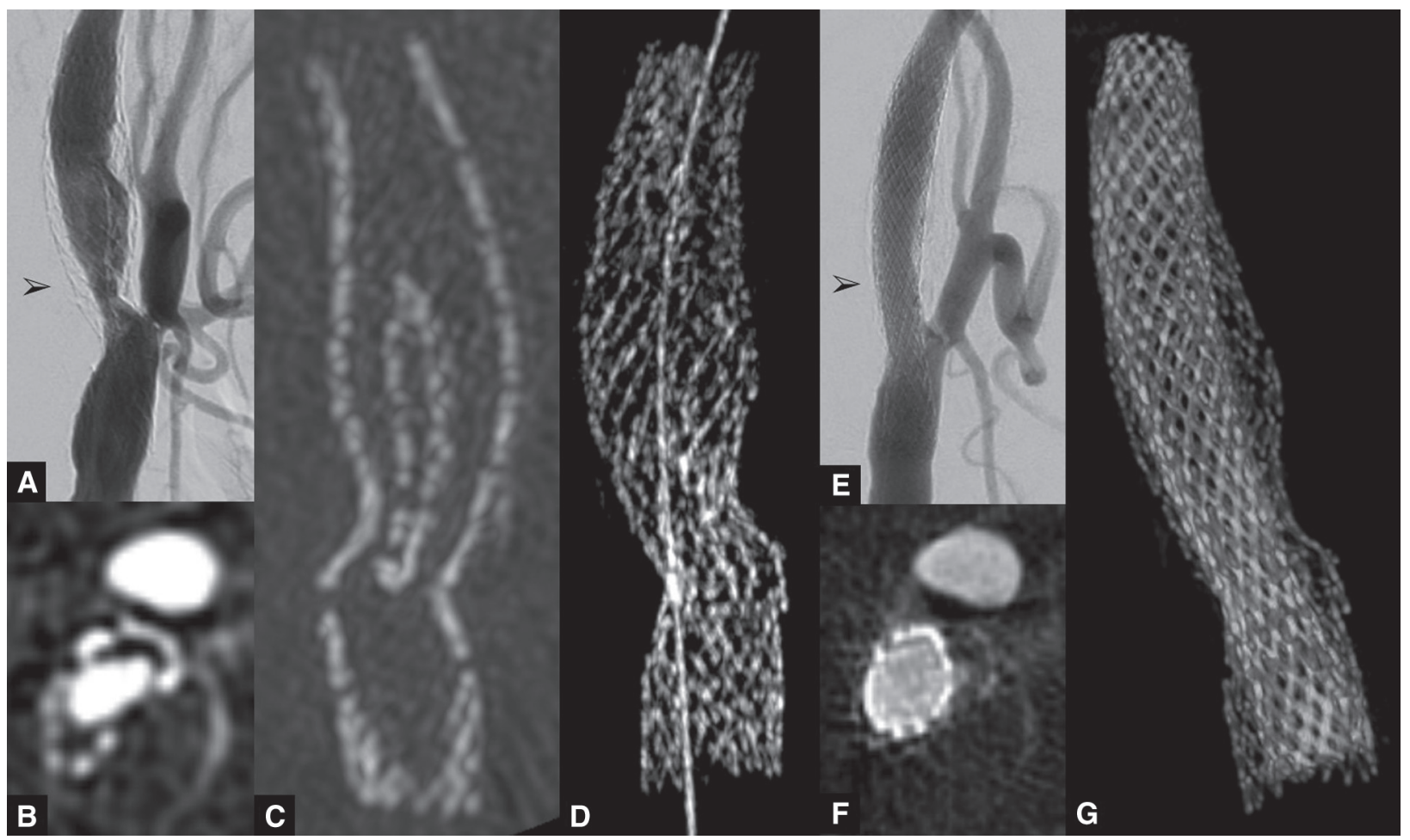

Fig. 3 On DSA (A), the stent lumen is markedly narrowed compared with that observed in Fig. 2B. Cone-beam CT (B) depicts the cross-section at the arrowhead (A). Illustration of the lateral view of a stent fracture at the site of calcification by CT (C). In-stent stenosis is effectively improved on postoperative angiography $(E)$. Conebeam CT $(\mathbf{F})$ depicts the cross-section at the arrowhead (E). A 3D view $(\mathbf{G})$ depicts the expansion of the narrowed lumen (D).

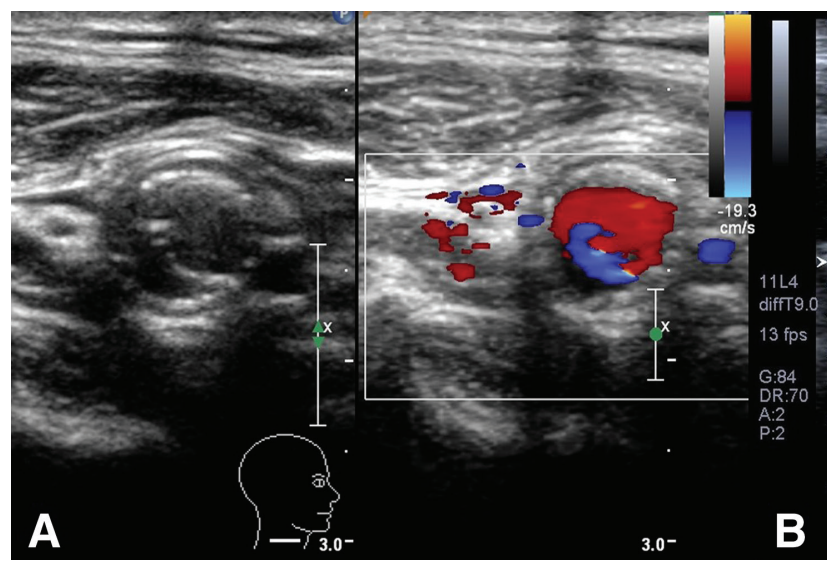

Fig. 4 The latest carotid artery duplex scan depicts an expanded lumen of the right carotid artery (A: a short-axis view, B: a

demonstrated the possibility of restenosis ${ }^{7}$ and stroke events. ${ }^{1)}$ The management of stent fracture or deformity is divided into observation with anti-platelet agents, ${ }^{1)}$ surgical removal, ${ }^{8)}$ and repeat CAS. ${ }^{2,3)}$ At first, close follow-up with dual antiplatelet therapy was chosen in the present case because stent fracture did not immediately cause carotid artery restenosis. Close follow-up revealed progression of the in-stent stenosis after 6 months due to

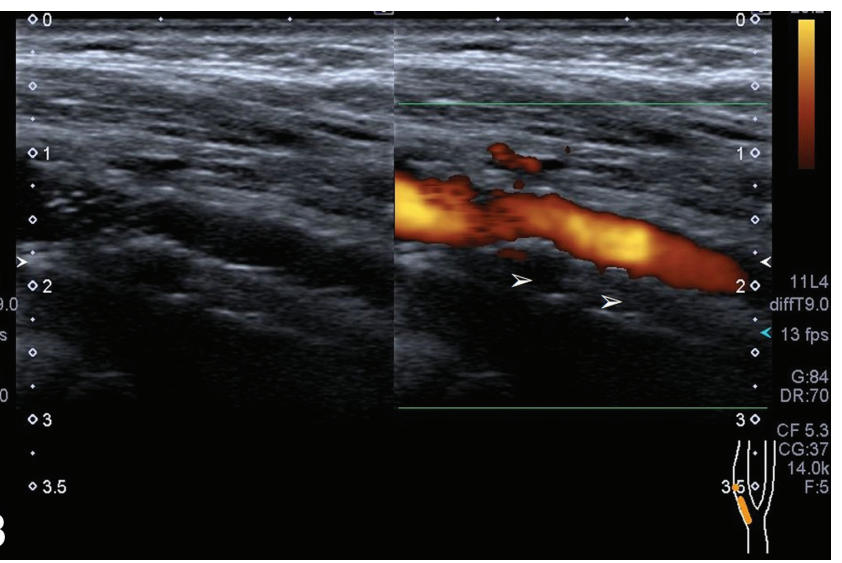

long-axis view, arrowheads: the space between Precise and Wallstent).

which repeat CAS was performed. To the best of our knowledge, this is the longest follow-up case report of repeat CAS for stent fracture.

A closed-cell stent is useful to expand the in-stent stenosis caused by open-cell stent fracture. Open-cell stents are more flexible than closed-cell stents because the freecell areas between struts are larger. ${ }^{9)}$ In spite of their flexibility, folding deformation of open-cell stents occur 
Fuse $Y$, et al.

when excessive pressure is on them. The deformation of PROTÉGÉ and PRECISE has been reported to establish at the site of calcification ${ }^{10,11)}$ or of a thick shaft of a protection device. ${ }^{12)}$ When the non-uniform pressure is on an open-cell strut, it tends to fold inwardly. In the current case, calcification was the risk of stent deformation, and post-dilation seems to have induced the deformation. On the other hand, the strut configuration of closed-cell stents may provide better scaffolding to vessels compared with open-cell stents. ${ }^{13)}$ Here, we place more emphasis on scaffolding than flexibility because the in-stent vessel lumen is relatively straight. Besides, the radial force was proved to be stronger in Wallstent than that in Precise or in Protégé. ${ }^{14)}$ With a stronger radial force, the better it seems for a stent to stand the compression during the operative procedure. Once an open-cell stent is fractured, it is reasonable to utilize a differently designed stent with balloon dilation to add a greater radial force.

The present case demonstrates the possibility of preventing future stroke events in patients with carotid artery stent fracture. In cases of stent deformity, close follow-up is necessary to detect progressive restenosis, which can be safely treated using a closed-cell stent placement. With the advent of new stent devices, further reports should be accumulated to determine the optimal stent device for re-stenting.

\section{Conclusion}

In conclusion, repeat CAS with a closed-cell stent is a viable treatment option for progressive in-stent stenosis caused by stent fracture.

\section{Acknowledgment}

The authors would like to thank Enago (www.enago.jp) for the English language review.

\section{Disclosure Statement}

The authors declare that they have no conflict of interest.

\section{References}

1) Nazzal M, Abbas J, Nazzal M, et al: Fractured internal carotid artery stent. Vascular 2008; 16: 179-182.
2) Ling AJ, Mwipatayi P, Gandhi T, et al: Stenting for carotid artery stenosis: fractures, proposed etiology and the need for surveillance. J Vasc Surg 2008; 47: 1220-1226; discussion 1226.

3) Abularrage CJ, Patel VI, Crawford RS, et al: Carotid stent fracture with recurrent high-grade stenosis. Ann Vasc Surg 2010; 24: 254.e11-e15.

4) Chang CK, Huded CP, Nolan BW, et al: Prevalence and clinical significance of stent fracture and deformation following carotid artery stenting. J Vasc Surg 2011; 54: 685-690.

5) Sfyroeras GS, Koutsiaris A, Karathanos C, et al: Clinical relevance and treatment of carotid stent fractures. $J$ Vasc Surg 2010; 51: 1280-1285.

6) Weinberg I, Beckman JA, Matsumura JS, et al: Carotid stent fractures are not associated with adverse events: results from the ACT-1 multicenter randomized trial (carotid angioplasty and stenting versus endarterectomy in asymptomatic subjects who are at standard risk for carotid endarterectomy with significant extracranial carotid stenotic disease). Circulation 2018; 137: 49-56.

7) Coppi G, Moratto R, Veronesi J, et al: Carotid artery stent fracture identification and clinical relevance. J Vasc Surg 2010; 51: 1397-1405.

8) Ross CB, Khoobehi A, Irwin CL, et al: Carotid artery stenting for stenosis following cervical radiotherapy: report of early failure with associated stent fracture. Vasc Endovascular Surg 2009; 43: 599-605.

9) Bosiers M, de Donato G, Deloose K, et al: Does free cell area influence the outcome in carotid artery stenting? Eur J Vasc Endovasc Surg 2007; 33: 135-141; discussion 142-143.

10) Naraoka M, Shimamura N, Matsuda N, et al: Stent folding deformation of PRECISE and PROTÉGÉ during carotid artery stenting: two case reports. JNET J Neuroendovasc Ther 2019; 13: 468-473.

11) Tetsuo $Y$, Matsumoto $H$, Nishiyama $H$, et al: A case of folding deformation of PROTÉGÉ stent during carotid artery stenting with distal embolic protection. JNET $J$ Neuroendovasc Ther 2018; 12: 131-135.

12) Murakami $T$, Shigematsu $T$, Kadono $Y$, et al: Stent folding deformation in carotid artery stenting with open-cell stent: a case report. JNET J Neuroendovasc Ther 2015; 9: 260-265.

13) Pierce DS, Rosero EB, Modrall JG, et al: Open-cell versus closed-cell stent design differences in blood flow velocities after carotid stenting. J Vasc Surg 2009; 49: 602-606; discussion 606.

14) Müller-Hülsbeck $S$, Schäfer PJ, Charalambous N, et al: Comparison of carotid stents: an in-vitro experiment focusing on stent design. J Endovasc Ther 2009; 16: 168-177. 